Article

\title{
Quasi-Icosahedral Clusters in Zr-Based Metallic Glasses
}

\author{
Guqing Guo \\ School of Applied Science, Taiyuan University of Science and Technology, Taiyuan 030024, China; \\ GuQingGuo@tyust.edu.cn; Tel.: +86-1391-448-8613
}

Received: 19 July 2020; Accepted: 19 August 2020; Published: 24 August 2020

\begin{abstract}
The icosahedral short-range order structure is one of the important local structural units in the field of metallic glasses. Based on the Voronoi tessellation method, the connection modes between shell atoms of Voronoi indexed clusters in $\mathrm{ZrCu}$ binary and $\mathrm{ZrCuAl}$ ternary metallic glasses were characterized systematically. It was revealed that Voronoi clusters with different connection modes can potentially have the same Voronoi index. By investigating the connection modes of clusters, three types of Voronoi indexed clusters can be defined as quasi-icosahedral clusters, which have the most similar five-fold environments to full icosahedral clusters. It was also found that the full icosahedral clusters and the quasi-icosahedral clusters had similar values for some structural parameters, and that these clusters contain almost all the atoms in these structural models, enhancing the space filling efficiency. Full icosahedral and quasi-icosahedral clusters should be regarded as the building blocks of atomic structures contributing to glass formation in amorphous alloys. This work is helpful for investigating and understanding the various macroscopic properties of metallic glasses at the atomic cluster level.
\end{abstract}

Keywords: icosahedral short-range order; icosahedral cluster; Voronoi tessellation; metallic glass; amorphous alloy; connection mode

\section{Introduction}

Metallic glasses (MGs) have attracted considerable attention because of their unique properties. The atomic structures of MGs are a fundamental and intriguing issue in condensed-matter physics, due to the importance of understanding their glass-forming mechanisms and unique properties [1,2]. So far, various simulations and experiments have been developed to reveal the microstructures of MGs [3-5]. Many structural models have been proposed over the years, such as the cluster dense packing model [6], the quasi-equivalent cluster close-packed model [7] and the global packing model [8]. In previous works [3-5,7], the icosahedral short-range order is usually detected when studying the microstructure of transition-metal (TM)-based MGs, an order that plays a key role in explaining the glass-forming ability (GFA) [8], supercooled liquid [9], elastic deformation [10] and so on.

In order to effectively characterize a given amorphous structure and extract the key structural features relevant to the fundamentals of glass formation and properties, many topological methods have been proposed to describe the structure of MGs-for example, the bond orientation order method [11], Honeycutt-pAnderson common neighbor analysis [12] and the Voronoi tessellation method. The Voronoi tessellation method $[4,5,13,14]$ is one of the widely used methods for analyzing short-range order icosahedral clusters. The full icosahedral structure [15] can be indexed as the $<0,0,12,0>$ cluster by the Voronoi tessellation method. However, many simulation and experimental works have shown that $\langle 0,0,12,0\rangle$ usually does not have a high concentration in many MG compositions [16] and is generally less than $10 \%$, or even less than $5 \%$ in some compositions $[17,18]$. Therefore, there is a major question: Is the concentration of icosahedral clusters actually very low in 
metallic glass structures, or is there a limitation of the Voronoi method when identifying icosahedral clusters? In order to address this key question, the typical binary TM MGs, $\mathrm{ZrCu}$, and ternary TM $\mathrm{MGs}, \mathrm{ZrCuAl}$, were selected as the target objects in this work. In previous works [4,15-20], various theoretical and experimental methods were used to research the structures of these kinds of MGs, which generally supposed that the cluster can be regarded as the structural unit, especially icosahedral clusters. The GFA and mechanical properties in metallic glass can be described from a structural perspective by analyzing the concentration and connection of clusters. In this work, based on the Voronoi tessellation method, a feasible scheme for identifying quasi-icosahedral clusters in MGs was developed, and it was found that full icosahedral and quasi-icosahedral clusters should be regarded as the building blocks contributing to glass formation in TM MGs.

\section{Materials and Methods}

In this work, the binary MGs $\mathrm{Zr}_{50} \mathrm{Cu}_{50}$ and the ternary MGs $\mathrm{Zr}_{48} \mathrm{Cu}_{45} \mathrm{Al}_{7}$ were selected as the samples, and their structures were characterized by the method of $\mathrm{X}$-ray diffraction. A synchrotron radiation experiment was used in a further step, and the results were then simulated by Reverse Monte Carlo (RMC) simulation to obtain the structural information from the level of atom to cluster. Details of the sample preparation method, synchrotron radiation experiment and RMC simulation can be found in [21,22]. The structural models that we simulated included 40,000 atoms that conformed to the stoichiometric ratios of individual samples. However, these two reliable amorphous structural models could not reflect the samples' structural characteristics. Thus, the structural models were analyzed by the Voronoi tessellation method. The Voronoi tessellation method divides space into polyhedrons around atoms by the construction of bisecting planes along the lines joining the central atom and all its neighbors. A Voronoi index $<\mathrm{n} 3, \mathrm{n} 4, \mathrm{n} 5, \mathrm{n} 6, \ldots>$ associated with the polyhedron is used to characterize the topology of the local atomic packing structure, where $n_{i}$ denotes the number of $i$-edged faces of the Voronoi polyhedron around the central atom or the connection between the shell atoms in the Voronoi cluster. By dividing the space into various polyhedrons centered at each atom, the Voronoi tessellation method classifies the local atomic structures according to the Voronoi index. $\Sigma n_{i}$ means the coordination number of the cluster. In this work, the Voronoi tessellation process was divided into two steps. The first step was to select the candidate neighbor atoms. One of the atoms was selected as the center atom, with $5 \AA$ as the cut-off radius for the first Voronoi tessellation. In constructing the Voronoi polyhedron, we removed those surfaces with an area of less than $2 \%$ of the total area of the polyhedron surfaces [7] and the corresponding candidate neighbor atoms in the Voronoi cluster were also removed. The second step was to obtain the final Voronoi cluster by performing the second Voronoi tessellation for the rest of candidate nearest neighbor atoms. All the subsequent analyses and discussion are based on the results obtained from the above steps.

\section{Results}

\subsection{Connection Mode Analysis}

By analyzing the Voronoi clusters in two samples, it is found that the shell atoms of clusters only have coordination numbers of 4,5 or 6 (number of connections with other shell atoms) while they do not have coordination number of 3, 7 or more, which is similar to the findings of other research works $[17,18]$. Thus, only the index style $\langle 0, n 4, n 5, n 6>$ is considered when discussing the Voronoi clusters.

Although the Voronoi tessellation method is mathematically rigorous and classifies the local atomic structures according to the Voronoi index, the connection modes between shell atoms are absent. Therefore, we referred to the stereographic projection method [23] to determine the connection modes between shell atoms. The projection method can transform the connections of shell atoms in three-dimensional space into a projection diagram on a two-dimensional plane. Figure 1a shows that we can choose any atom in the objective cluster as the projection plane. The point " $\mathrm{P}$ " is a projection 
pole that connects the spherical centers of shell atoms and cluster centers as a collinear, and the atom on the top of cluster is projected as a disc onto the two-dimensional plane, as we can see in Figure $1 \mathrm{~b}$. The rest of the shell atoms and the connection relations on the disc are projected in order to obtain Figure 1c. Figure 1d shows the final projection plane after abstracting the coordination numbers and connection modes.

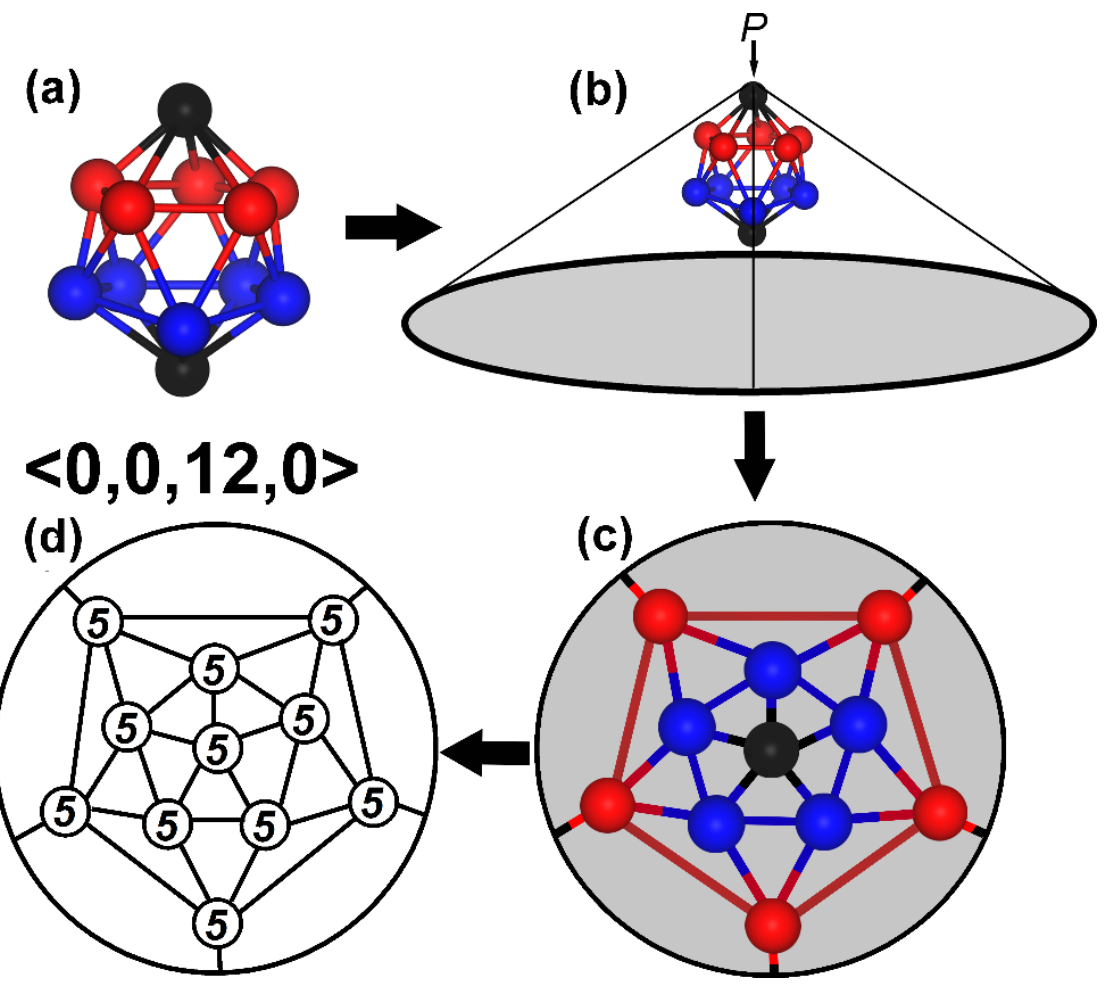

Figure 1. Schematic diagram of the projection method for reflecting the connection modes between shell atoms based on Voronoi indexed clusters. (a) Voronoi cluster $\langle 0,0,12,0\rangle$ is selected as the studied prototype; (b) The shell atom on the top of cluster is projected as a disc onto the two-dimensional plane from projection pole point " $\mathrm{P}$ "; (c) The rest of the shell atoms and the connection relations are projected onto the two-dimensional plane; (d) The final projection plane after abstracting connection modes, and the number 5 represents the coordination number.

In this work, we calibrated the projection relations of all clusters in the $\mathrm{ZrCu}$ binary and $\mathrm{ZrCuAl}$ ternary MG structural models, then analyzed all possible models of Voronoi clusters. Figure 2a shows the two-dimensional connection projection graph of the $<0,0,12,0>$ cluster. Each line shows a bond between two shell atoms in the cluster. The $<0,0,12,0>$ cluster model has only one projection style due to its regular structure. However, the Voronoi clusters indexed $<0,2,8,2>$ and $<0,4,4,4>$ have two and five projection models, respectively, as shown in Figure 2c-h. Comparing the clusters $<0,0,12,0\rangle$ and $<0,2,8,2>-\mathrm{I}$, the blue line and dotted line show the difference between the two clusters, which means that the $<0,0,12,0\rangle$ cluster can transform into $<0,2,8,2\rangle$ in three-dimensional space when the bond between a pair of atoms on the shell of the $<0,0,12,0>$ cluster changes. Similarly, when the connection between a pair of atoms changes in $<0,2,8,2>-\mathrm{I}$ (in principle, a change in coordination number would not form three nor seven or more coordination shell atoms), three kinds of clusters, $<0,2,8,2>-\mathrm{I},<0,4,4,4>-\mathrm{I}$ and $<0,4,4,4>-\mathrm{II}$, will be obtained thereafter. The red line and dotted line show the difference between these three clusters. By analyzing all the simulations, we conclude that, if there are $\mathrm{n}_{4}$ and $\mathrm{n}_{6}$, and the total coordination number in the cluster is higher, the cluster will have more projection structures, and thus is a so-called "disordered cluster". Meanwhile, we observed that a difference exists in only one pair of atoms between $<0,2,8,2>-\mathrm{I}$, II clusters and $<0,4,4,4>-\mathrm{I}$, II clusters, which means that these clusters have a similar structure. However, the other atomic structure of the 
$<0,4,4,4>$ cluster has more differences in terms of the I, II connection modes. Therefore, there are still some limitations to using the Voronoi index to classify clusters due to their different connection modes, as in the case of the $<0,4,4,4>$ cluster. However, the $<0,0,12,0>$ cluster has only one configuration, which means that the Voronoi index can classify the space structure well. Table 1 shows the Voronoi clusters with a single configuration and with a total coordination number in the range of 9-14.

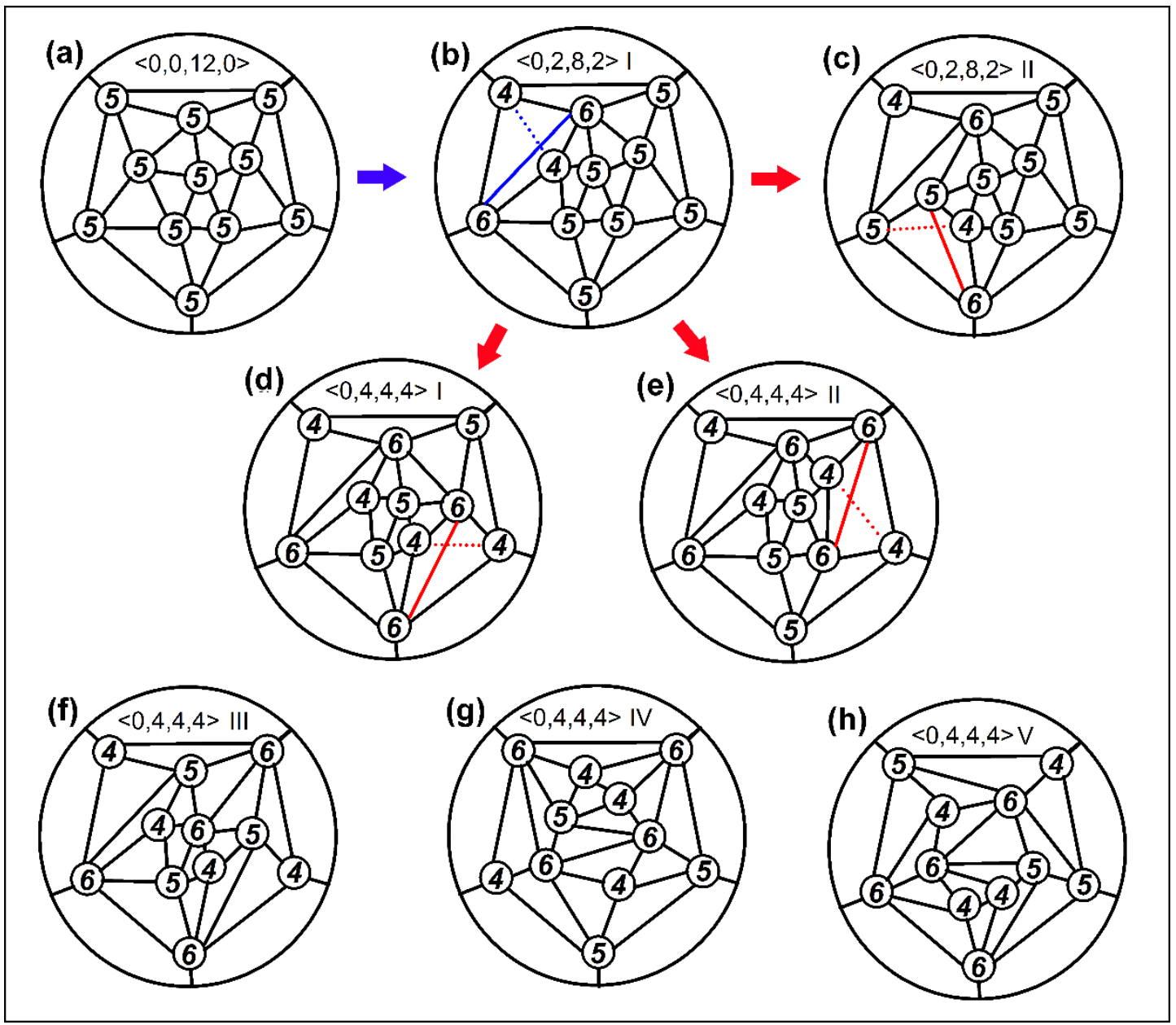

Figure 2. Planar projection reflecting the shell atom connection modes of Voronoi indexed clusters. (a) The planar projection of $<0,0,12,0\rangle$; (b), (c) Two types of planar projection of $<0,2,8,2\rangle$; (d)-(h) Five types of planar projection of $<0,4,4,4>$.

Table 1. Voronoi clusters with only one type of planar projection.

\begin{tabular}{ccccccc}
\hline $\begin{array}{c}\text { Coordination } \\
\text { Number }\end{array}$ & $\mathbf{9}$ & $\mathbf{1 0}$ & $\mathbf{1 1}$ & $\mathbf{1 2}$ & $\mathbf{1 3}$ & $\mathbf{1 4}$ \\
\hline $\begin{array}{c}\text { Voronoi } \\
\text { Index }\end{array}$ & $\begin{array}{l}<0,3,6,0> \\
<0,4,4,1>\end{array}$ & $\begin{array}{l}<0,2,8,0> \\
<0,3,6,1>\end{array}$ & $<0,2,8,1>$ & $<0,0,12,0>$ & $<0,1,10,2>$ & $<0,0,12,2>$ \\
\hline
\end{tabular}

\subsection{Quasi-Icosahedral Clusters}

The full icosahedral cluster with a complete five-fold environment has a Voronoi index of $<0,0,12,0>$. In the full icosahedral cluster, each shell atom bonds with five other shell atoms, demonstrating a complete five-fold environment [15]. However, in many experiments and simulations, amorphous alloys with relatively disordered atomic arrangements contain lower contents of the $<0,0,12,0\rangle$ cluster. The content was only $5 \%$ in the $\mathrm{ZrCu}$ samples that we prepared. Fortunately, in previous works, it was found that the concentration of Voronoi clusters that have an incomplete five-fold environment but 
have a similar configuration to the $<0,0,12,0>$ cluster is as high as $30 \%[15,16]$. Thus, in this work, we focused on the determination of quasi-icosahedral clusters. With reference to the $\langle 0,0,12,0\rangle$ cluster, three deformation modes were adopted to identify quasi-icosahedral clusters using the projection method: (1) change the connection between one pair of atoms in the $\langle 0,0,12,0\rangle$ cluster; (2) remove a shell atom from the $\langle 0,0,12,0\rangle$ cluster; (3) add an atom to the $\langle 0,0,12,0\rangle$ cluster. As shown in Figure 3 , the configurations of the three clusters $<0,2,8,2>-\mathrm{I},<0,2,8,1>$ and $<0,1,10,2>$ can be obtained by the method described above. Furthermore, it was revealed that these three clusters' configurations simply involve local connection modes that are distorted when compared with the $<0,0,12,0\rangle$ cluster, as shown by the red dotted line in Figure 3. These three quasi-icosahedral clusters do not only have high five-fold symmetry (the $<0,1,10,2>$ cluster reflects 10 five-fold symmetry structures), but also retain most of the features of the $\langle 0,0,12,0\rangle$ cluster in terms of connection modes of shell atoms, which fully show that the configurations of these three clusters are highly similar to the $<0,0,12,0\rangle$ cluster. Therefore, they can be defined as quasi-icosahedral clusters.

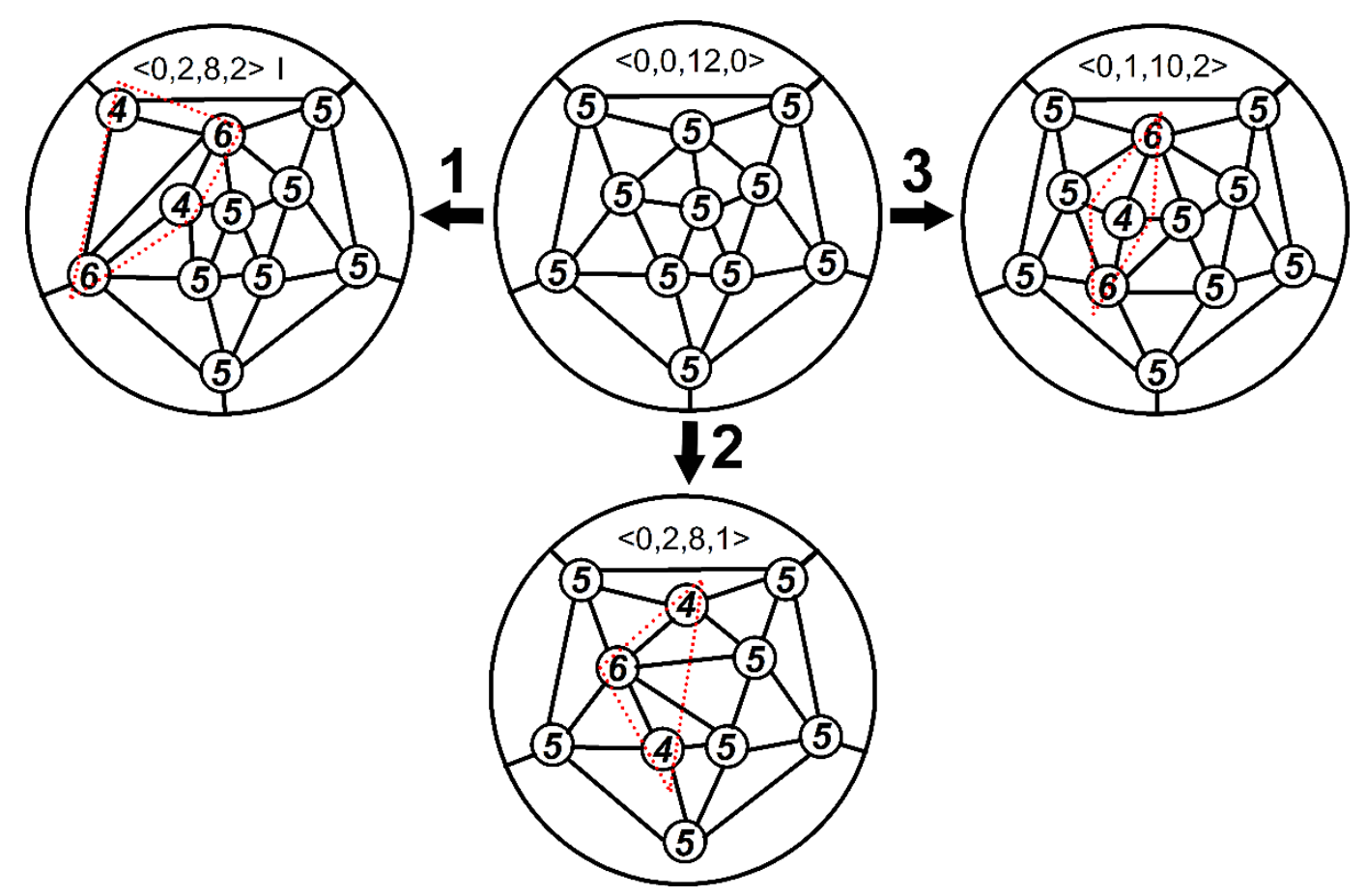

Figure 3. Planar projections of the full icosahedral cluster $\langle 0,0,12,0\rangle$ and three corresponding clusters transformed from $<0,0,12,0>$.

For deformation model 1, if two pairs of bonding mode change, $<0,2,8,2>$-II or $<0,4,4,4>$ I, II clusters can be obtained. Figure $2 c-e$ shows that there are at least two local five-fold symmetry structures of the above clusters that have been destroyed, and the overall shell atom connections have been greatly changed when compared with the $\langle 0,0,12,0\rangle$ cluster. This result means that the above clusters cannot be defined as quasi-icosahedral clusters because of their much more "distorted or incomplete" icosahedral structure. However, for deformation models 2 and 3, since the MGs include at least two elements that have different atomic radii, according to the cluster dense packing model [6,24], the coordination number of clusters centered around an atom with a small (big) radius is preferentially less (more) than 12. Therefore, while it is reasonable to expect 12-coordination clusters, there are a lot of non-12-coordination clusters in MGs. Based on our selection criteria, clusters indexed $<0,0,12,0>,<0,2,8,1>$ (coordination number is 11 ), $<0,1,10,2>$ (coordination number is 11 ) or $<0,2,8,2>$ (coordination number is 12) can be regarded as icosahedral clusters. Both $\langle 0,2,8,1\rangle$ and $\langle 0,1,10,2\rangle$ are non-12-coordination clusters, which means that non-12-coordination quasi-icosahedral clusters could be abundant in MG structural models. 


\subsection{Quasi-Icosahedral Clusters in Zr-Based MGs}

To further understand the relationship between quasi-icosahedral clusters and the formation of amorphous alloys, some structural parameters that have relatively strong relationships with the GFA of MGs were studied [5]. We extracted the quasi-icosahedral clusters centered around a $\mathrm{Zr}, \mathrm{Cu}$, or $\mathrm{Al}$ atom from the samples and made calculations for $D$ (atomic packing efficiency) and $T$ (cluster regularity; the closer the value of $\mathrm{T}$ is to 0 , the more regular the cluster is). Table 2 shows the calculation values. As a contrast, the values of mean $D$ and $T$ for all clusters of the structural models were calculated too. The values show that both the full icosahedral and quasi-icosahedral clusters have similar values for these structural parameters, which fully illustrates that quasi-icosahedral clusters have the most similar five-fold environment to full icosahedral clusters. Moreover, compared with the mean values of all clusters, icosahedral clusters have better structural features in terms of high atomic packing efficiency, high cluster regularity and a fruitful five-fold environment. The results indicate that quasi-icosahedral clusters can make an important structural contribution to the formation of amorphous alloys.

Table 2. Structural information of clusters.

\begin{tabular}{|c|c|c|c|c|c|c|c|}
\hline & \multirow{2}{*}{ Samples } & \multicolumn{2}{|c|}{ Zr-Centered } & \multicolumn{2}{|c|}{ Cu-Centered } & \multicolumn{2}{|c|}{ Al-Centered } \\
\hline & & $D(\%)$ & $T$ & $D(\%)$ & $T$ & $D(\%)$ & $T$ \\
\hline \multirow{3}{*}{$\mathrm{Zr}_{50} \mathrm{Cu}_{50}$} & $<0,0,12,0>$ & 71.99 & 0.0284 & 67.63 & 0.0290 & - & - \\
\hline & Quasi-icosahedral clusters & 71.05 & 0.0326 & 67.51 & 0.0359 & - & - \\
\hline & All Voronoi clusters & 70.19 & 0.0349 & 67.28 & 0.0419 & - & - \\
\hline \multirow{3}{*}{$\mathrm{Zr}_{48} \mathrm{Cu}_{45} \mathrm{Al}_{7}$} & $<0,0,12,0>$ & 72.21 & 0.0278 & 67.93 & 0.0294 & 75.86 & 0.0204 \\
\hline & Quasi-icosahedral clusters & 71.44 & 0.0313 & 67.84 & 0.0354 & 75.35 & 0.0274 \\
\hline & All Voronoi clusters & 70.42 & 0.0325 & 67.26 & 0.0416 & 74.96 & 0.0299 \\
\hline
\end{tabular}

To obtain more information about quasi-icosahedral clusters in different components, the distribution of the main clusters was studied, as shown in Figure 4. Figure 4a shows the contents of Voronoi clusters over 1\% in two MG samples, where we can find that the contents of quasi-icosahedral clusters $<0,2,8,1>,<0,1,10,2>$ and $<0,2,8,2>-$-I are generally higher, which means that these three configurations of clusters are major units in the MG samples. Furthermore, the proportion of the center atoms in the abovementioned quasi-icosahedral clusters and the proportion of center and shell atoms out of the total number of atoms (double-counted atoms were counted only once) were also calculated, and the results are shown in Figure $4 \mathrm{~b}$. The full icosahedral and quasi-icosahedral clusters in $\mathrm{Zr}_{50} \mathrm{Cu}_{50}$ and $\mathrm{Zr}_{48} \mathrm{Cu}_{45} \mathrm{Al}_{7}$ account for $28.5 \%$ and $30.1 \%$, respectively. Moreover, the atoms in the full icosahedral and quasi-icosahedral clusters account for over $96 \%$ of the total atoms in the samples, at $96.8 \%$ and $99.8 \%$, respectively. The results show that the full icosahedral and quasi-icosahedral clusters, which includes almost all atoms in the structural model, can be regarded as the main units in MGs. Therefore, the central atoms of icosahedral clusters can be regarded as solute atoms without distinguishing their elemental differences. This result makes our method more reasonable for studying the microstructure in MG systems. Furthermore, the concept can also be applied to multicomponent alloys in which the components cannot be divided into solutes and solvents easily, such as high-entropy alloys. 

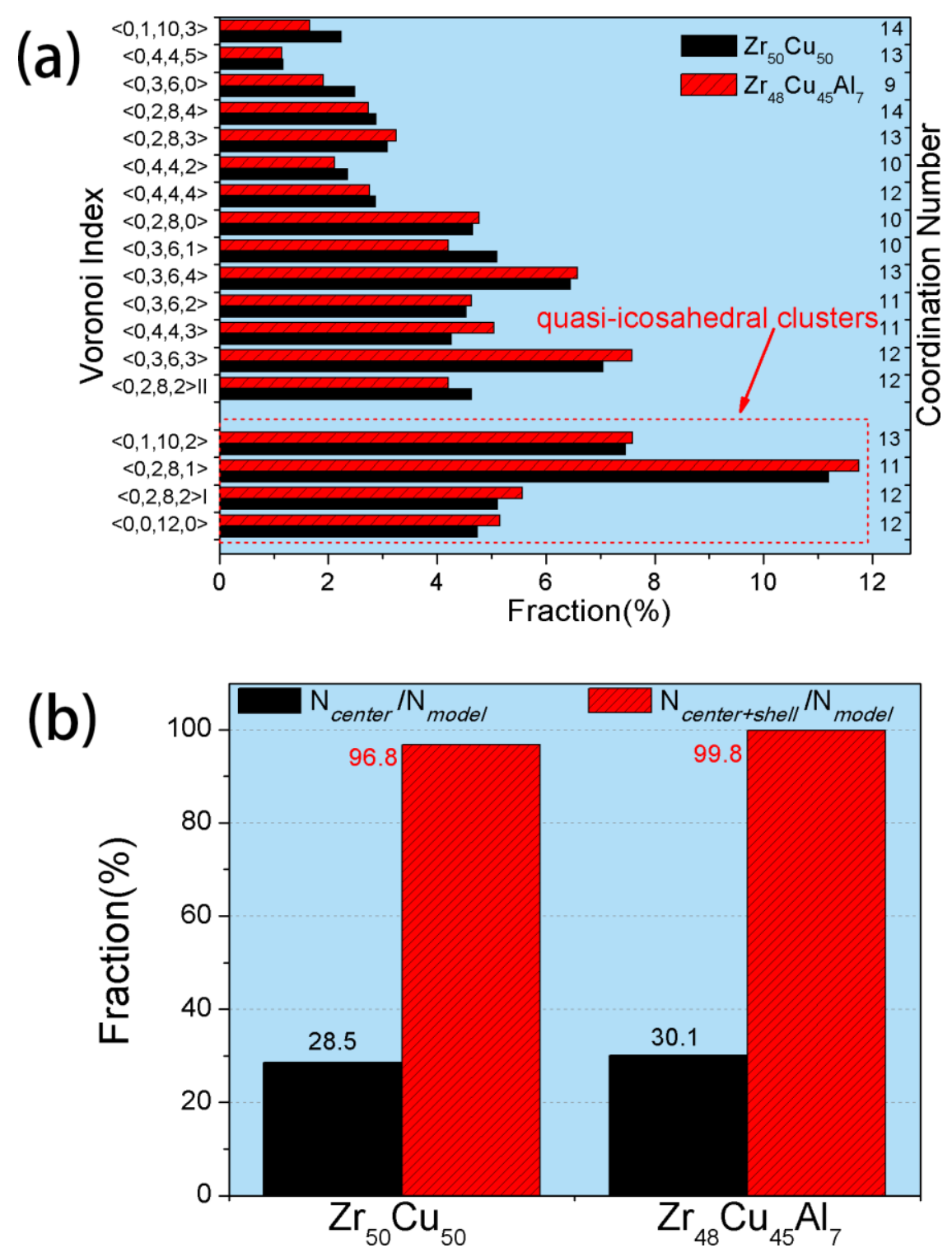

Figure 4. (a) Distribution of major Voronoi clusters; (b) the proportions of center atoms (center and shell atoms) in all the quasi-icosahedral clusters in the structural model. $\mathrm{N}_{c e n t e r}$ is the number of center atoms in the quasi-icosahedral clusters, $\mathrm{N}_{\text {center }+ \text { shell }}$ is the total number of atoms contained in the quasi-icosahedral clusters and $\mathrm{N}_{\text {model }}$ is the total number of atoms in the structural model.

Full icosahedral clusters cannot fulfill 3-D space tiling because they are quasi-periodic. The icosahedral clusters, as the main structures with high structural stability, are bonded with each other by interpenetrating connections, and form triangle, double triangle, triple triangle, tetrahedron and chain connected icosahedral medium-range order clusters [3]. Therefore, compared with the full icosahedron arrangement in quasi-crystals, our modes are more flexible. The clusters include not only 12-coordination icosahedral clusters, but also $11-(<0,2,8,1>)$ and $13-(<0,1,10,2>)$ coordination quasi-icosahedral clusters. Relative to the arrangement of strictly icosahedral clusters, connecting the 11-, 12- and 13-coordination quasi-icosahedral clusters can increase the cluster connection ability and the space filling rate of stacking. In addition, we found that almost all atoms are contained in various kinds of clusters in this work. However, there is still a small number of atoms that exists between clusters for connecting and stabilizing neighbor clusters—-that is, the so-called "glue atoms effect" $[25,26]$. 


\section{Conclusions}

In this work, based on the Voronoi tessellation method, we proposed a feasible scheme for identifying quasi-icosahedral clusters in MGs. Three Voronoi clusters-namely, $\langle 0,2,8,1\rangle,\langle 0,1,10,2\rangle$ and $<0,2,8,2>-\mathrm{I}$, were extracted by this method. Compared with other clusters in the MG structural model, the structure of these three quasi-icosahedral clusters is similar to that of full icosahedral clusters, and they are popular structural units in amorphous structures. Moreover, these three quasi-icosahedral clusters have high atomic packing efficiency, cluster regularity and five-fold symmetry, which is similar to full icosahedral clusters. We also found that the center atom of a quasi-icosahedral cluster can be regarded as the solute atom in the cluster model of MGs, which contained almost all of the atoms for stabilizing the basic structure of MGs. Thus, full icosahedral and quasi-icosahedral clusters should be regarded as the main structural units of MGs, and they make an important structural contribution to the formation of amorphous alloys. These results are helpful for investigating and understanding various macroscopic properties of MGs at the atomic cluster level.

Funding: This research was funded by Scientific and Technological Innovation Programs of Higher Education Institutions in Shanxi, grant number 2019L0612, and Taiyuan University of Science and Technology Scientific Research Initial Funding, grant number 20162039.

Conflicts of Interest: The author declares no conflict of interest.

\section{References}

1. Inoue, A. Stabilization of metallic supercooled liquid and bulk amorphous alloys. Acta Mater. 2000, 48, 279-306. [CrossRef]

2. Wu, Z.W.; Li, M.Z.; Wang, W.H.; Liu, K.X. Hidden topological order and its correlation with glass-forming ability in metallic glasses. Nat. Commun. 2015, 6, 6035. [CrossRef] [PubMed]

3. Ren, L.; Gao, T.H.; Ma, R.; Xie, Q.; Tian, Z.; Chen, Q.; Liang, Y.C.; Hu, X.C. The connection of icosahedral and defective icosahedral clusters in medium-range order structures of CuZrAl alloy. J. Non-Cryst. Solids 2019, 512, 119475. [CrossRef]

4. Cheng, Y.Q.; Ma, E. Atomic-level structure and structure-property relationship in metallic glasses. Prog. Mater. Sci. 2011, 56, 379-473. [CrossRef]

5. Yang, L.; Guo, G.Q.; Chen, L.Y.; Huang, C.L.; Ge, T.; Chen, D.; Liaw, P.K.; Saksl, K.; Ren, Y.; Zeng, Q.S.; et al. Atomic-Scale Mechanisms of the Glass-Forming Ability in Metallic Glasses. Phys. Rev. Lett. 2012, 109, 105502. [CrossRef] [PubMed]

6. Miracle, D.B. A structural model for metallic glasses. Nat. Mater. 2004, 3, 697-702. [CrossRef]

7. Sheng, H.W.; Luo, W.K.; Alamgir, F.M.; Bai, J.M.; Ma, E. Atomic packing and short-to-medium-range order in metallic glasses. Nature 2006, 439, 419-425. [CrossRef]

8. Liu, X.J.; Xu, Y.; Hui, X.; Lu, Z.P.; Li, F.; Chen, G.L.; Lu, J.; Liu, C.T. Metallic Liquids and Glasses: Atomic Order and Global Packing. Phys. Rev. Lett. 2010, 105, 155501. [CrossRef]

9. Schenk, T.; Holland, M.D.; Simonet, V.; Bellissent, R.; Herlach, D.M. Icosahedral short-range order in deeply undercooled metallic melts. Phys. Rev. Lett. 2002, 89, 075507. [CrossRef]

10. Wakeda, M.; Shibutani, Y. Icosahedral clustering with medium-range order and local elastic properties of amorphous metals. Acta Mater. 2010, 58, 3963-3969. [CrossRef]

11. Steinhardt, P.J.; Nelson, D.R.; Ronchetti, M. Bond-orientational order in liquids and glasses. Phys. Rev. B 1983, 28, 784-805. [CrossRef]

12. Honeycutt, J.D.; Andersen, H.C. Molecular-dynamics study of melting and freezing of small lennard-jones clusters. J. Phys. Chem. 1987, 91, 4950-4963. [CrossRef]

13. Finney, J.L. Modeling structures of amorphous metals and alloys. Nature 1977, 266, 309-314. [CrossRef]

14. Finney, J.L. Random packings and structure of simple liquids 2. Molecular geometry of simple liquids. Proc. R. Soc. Ser. A 1970, 319, 479-495.

15. Cheng, Y.Q.; Ma, E.; Sheng, H.W. Atomic level structure in multicomponent bulk metallic glass. Phys. Rev. Lett. 2009, 102, 245501. [CrossRef]

16. Yang, L.; Guo, G.Q. Preferred clusters in metallic glasses. Chin. Phys. B 2010, 19, 126101. [CrossRef] 
17. Li, M.Z.; Wang, C.Z.; Hao, S.G.; Kramer, M.J.; Ho, K.M. Structural heterogeneity and medium-range order in $\mathrm{Zr}_{\mathrm{x}} \mathrm{Cu}_{100-\mathrm{x}}$ metallic glasses. Phys. Rev. B 2009, 80, 184201. [CrossRef]

18. Fujita, T.; Konno, K.; Zhang, W.; Kumar, V.; Matsuura, M.; Inoue, A.; Sakurai, T.; Chen, M.W. Atomic-scale heterogeneity of a multicomponent bulk metallic glass with excellent glass forming ability. Phys. Rev. Lett. 2009, 103, 075502. [CrossRef]

19. Wang, S.Y.; Kramer, M.J.; Xu, M.; Wu, S.; Hao, S.G.; Sordelet, D.J.; Ho, K.M.; Wang, C.Z. Experimental and ab initio molecular dynamics simulation studies of liquid $\mathrm{Al}_{60} \mathrm{Cu}_{40}$ alloy. Phys. Rev. B 2009, $79,144205$. [CrossRef]

20. Hao, S.G.; Wang, C.Z.; Kramer, M.J.; Ho, K.M. Microscopic origin of slow dynamics at the good glass forming composition range in $\mathrm{Zr}_{1-\mathrm{x}} \mathrm{Cu}_{\mathrm{x}}$ metallic liquids. J. Appl. Phys. 2010, 107, 053511. [CrossRef]

21. Yang, L.; Guo, G.Q.; Chen, L.Y.; Wei, S.H.; Jiang, J.Z.; Wang, X.D. Atomic structure in Al-doped multicomponent bulk metallic glass. Scr. Mater. 2010, 63, 879-882. [CrossRef]

22. Yang, L.; Guo, G.Q.; Zhang, G.Q. Atomic structure of $\mathrm{Zr}_{48} \mathrm{Cu}_{45} \mathrm{Al}_{7}$ bulk metallic glass. Acta Phys. Sin. 2011, 60, 016103.

23. Stereographic Projection. Available online: http://encyclopediaofmath.org/index.php?title=Stereographic_ projection\&oldid=32504 (accessed on 19 August 2020).

24. Miracle, D.B. The efficient cluster packing model-An atomic structural model for metallic glasses. Acta Mater. 2006, 54, 4317-4336. [CrossRef]

25. Yang, L.; Xia, J.H.; Wang, Q.; Dong, C.; Chen, L.Y.; Ou, X.; Liu, J.F.; Jiang, J.Z.; Klementiev, K.; Saksl, K.; et al. Design of $\mathrm{Cu}_{8} \mathrm{Zr}_{5}$-based bulk metallic glasses. Appl. Phys. Lett. 2006, 88, 241913. [CrossRef]

26. Xia, J.H.; Qiang, J.B.; Wang, Y.M.; Wang, Q.; Dong, C. Ternary bulk metallic glasses formed by minor alloying of $\mathrm{Cu}_{8} \mathrm{Zr}_{5}$ icosahedron. Appl. Phys. Lett. 2006, 88, 10. [CrossRef]

(C) 2020 by the author. Licensee MDPI, Basel, Switzerland. This article is an open access article distributed under the terms and conditions of the Creative Commons Attribution (CC BY) license (http://creativecommons.org/licenses/by/4.0/). 